identified to act as a point of contact should the participant lose capacity to consent to further assessments. Once recruited to the study the researcher ensured regular contact with both participant and consultee throughout their inpatient stay.

Results A total of 101 people have participated in these studies over a 2 -year period. The high recruitment rate $(83 \%$, $n=101 / 121$ ) demonstrates a willingness of the hospice to take part in end of life research; this is consistent with previous work about palliative care research participation. Feedback has been positive with patients, caregivers and staff through the entire research process.

Conclusions The advance consent process is an effective way to facilitate research participation at the end of life. People with palliative care needs want to participate in studies which include assessments in the dying phase of their illness. Caregivers have shown great desire to support research. Researchers should consider the potential to use the advance consent process to improve research participation for people who are dying.

\section{HOME PARENTAL NUTRITION FOR PALLIATIVE PATIENTS. A REVIEW OF FIVE YEARS OF EXPERIENCE AT MILTON KEYNES UNIVERSITY HOSPITAL}

Jane Wale, Lynfa Lanzon-Miller, Marrison Marwood. Milton Keynes University Hospital, University of Buckingham Medical School

\subsection{6/spcare-2020-PCC.115}

Background Parenteral Nutrition (PN) is the intravenous administration of nutrition and can be used in patients whose gastrointestinal tract is unable to function. It is a treatment that is relatively easy to initiate, but it is a difficult treatment to continue outside of hospital and can also be, ethically and morally, a difficult treatment to stop. Recent ESPEN guidelines (2016) state that 'the bioethical aspects of feeding patients with advanced disease should be considered.... There are data showing benefits of home artificial nutrition in cancer patients .... even in advanced cancer patients. Home parenteral nutrition is a complex therapy and selecting patients for this treatment a demanding task'.

Method Patients for whom home parenteral nutrition had been a goal were identified. A retrospective review of these patients‘ clinical records was undertaken.

Results Between 2014 and 2019 six palliative patients had PN commenced at Milton Keynes University Hospital, a medium sized District General Hospital. One further patient had PN started at a tertiary centre. All patients had ongoing bowel obstruction secondary to peritoneal metastases. The mean age of patients was 49 years (range 35-81 years). The average time from starting PN to hospital discharge was 47 days for the MKUH patients compared to 14 days for the patient who was started on PN at a tertiary centre. The average time from staring PN to death was 435 days (range 28 - 1825 days). One patient remains alive, 2 died in the hospital, 2 in the hospice, 2 at home.

Conclusion Carefully selected palliative patients can benefit from home PN, but in a district general hospital this requires a lengthy hospital admission to achieve. The decision to start PN should be multidisciplinary and the goals of treatment should be clearly defined and reviewed regularly.
Global palliative care | posters $96-101$

\section{UNDERSTANDING THE MEANING ATTRIBUTED BY JORDANIAN PARENTS OF CHILDREN WITH CANCER TO THEIR ILLNESS: A PHENOMENOLOGICAL STUDY}

Maha Atout, Abd Al-Hadi Hasan, Rabia Allari, Amani Alkharabsheh, Mahmoud Thalgi, Noureen Shivji. Philadelphia University

\subsection{6/spcare-2020-PCC.116}

Background Jordanian culture is a variant of Arab-Islamic culture that comprises two intertwined strands: the interpretation of Islamic religion and vernacular Arab culture. Few studies have been conducted in the Middle East to explain the way culture shapes and reflects parents' lived experiences and interactions with their children, particularly concerning the meaning and philosophy of disease.

Methods The aim of this study was to explore the lived experiences of Jordanian parents of children with cancer. The study investigated the cultural meaning of illness and how it affected parents' lived experiences of their children's illness. The study adopted a hermeneutic phenomenological approach informed by the philosophy of Martin Heidegger and used semi-structured interviews. The study was conducted in one paediatric oncology unit in one Jordanian hospital.

Results Twenty-five interviews were collected from 24 mothers and one father. During the interviews, the parents expressed that their spiritual beliefs helped them accept their child's disease. Showing patience towards the suffering of their children was perceived as an important value the parents tried to stick to despite the deep suffering the children were experiencing. All the parents demonstrated a thankful approach as they perceived that everything that happens to their child is according to destiny. The beliefs the parents held about their child's illness were perceived to positively affect the way they interacted with them. This was especially so in sensitive situations, such as their reactions to initial diagnoses and receiving bad news.

Conclusion The findings of this study will inform the education of health professionals and increase their understanding of the beliefs and practices demonstrated by the study's participants and their integration into supportive patient plans.

\section{HOW DO PHYSICIANS IN SOUTH INDIA RECOGNISE, ASSESS AND MANAGE CHRONIC BREATHLESSNESS AND IS CHRONIC BREATHLESSNESS CONSIDERED A SYNDROME OR DIAGNOSIS?}

Matilda MM Barnes-Harris, Miriam J Johnson, Sunitha Daniel, Venkateswaran Chitra. Hull York Medical School, Wolfson Palliative Care Research Centre, University of Hull, Amrita Institute of Medical Sciences Amrita University

\subsection{6/spcare-2020-PCC.117}

Background Chronic breathlessness syndrome has been recently defined to help clinicians actively seek and patients legitimately present with persistent breathlessness, and to drive service development and research. There are many evidence-based interventions emerging. However, the naming and defining process did not include views from low to middle-income countries, where chronic breathlessness is a significant issue. 